\title{
Evaluation of the impact of an integrated care pathway for pulmonary embolism: a quasi-experimental pre-post study
}

\author{
Nuria Rodríguez-Núñez ${ }^{1}$, Alberto Ruano-Raviña ${ }^{2,3,4}$, Adriana Lama ${ }^{1}$, Lucía Ferreiro ${ }^{1,5}$, \\ Jorge Ricoy ${ }^{1}$, José M. Álvarez-Dobaño ${ }^{1,5}$, Juan Suárez-Antelo ${ }^{1}$, M. Elena Toubes ${ }^{1}$, Carlos Rábade ${ }^{1}$, \\ Antonio Golpe ${ }^{1,5}$, Vanessa Riveiro ${ }^{1}$, Ana Casal ${ }^{1}$, Romina Abelleira ${ }^{1}$, \\ Francisco Javier González-Barcala ${ }^{1,5}$, José R. González-Juanatey ${ }^{6}$, Luis Valdés ${ }^{1,5}$
}

${ }^{1}$ Department of Pulmonology, Complejo Hospitalario Universitario de Santiago, Santiago de Compostela, Spain; ${ }^{2}$ Department of Preventive Medicine and Public Health, Universidad de Santiago de Compostela, Santiago de Compostela, Spain; ${ }^{3}$ CIBER of Epidemiology and Public Health, CIBERESP, Madrid, Spain; ${ }^{4}$ Group C013, Health Research Institute of Santiago de Compostela (IDIS), Santiago de Compostela, Spain; ${ }^{5}$ Interdisciplinary Research Group on Pulmonology, Instituto de Investigaciones Sanitarias de Santiago (IDIS), Santiago de Compostela, Spain; ${ }^{6}$ Service of Cardiology, Spanish Network-Center for Cardiovascular Biomedical Research (CIBERCV), Health Research Institute of Santiago de Compostela (IDIS), Santiago de Compostela, Spain

Contributions: (I) Conception and design: N Rodríguez-Núñez, A Ruano-Raviña, L Valdés; (II) Administrative support: N Rodríguez-Núñez, A Ruano-Raviña; (III) Provision of study materials or patients: N Rodríguez-Núñez, A Lama, L Ferreiro, J Ricoy, JM Álvarez-Dobaño, J SuárezAntelo, ME Toubes, C Rábade, A Golpe, FJ González-Barcala; (IV) Collection and assembly of data: N Rodríguez-Nuñez, A Ruano-Raviña, L Valdés; (V) Data analysis and interpretation: N Rodríguez-Nuñez, A Ruano-Raviña, L Valdés; (VI) Manuscript writing: All authors; (VII) Final approval of manuscript: All authors.

Correspondence to: Prof. Dr. Alberto Ruano-Raviña. Department of Preventive Medicine and Public Health, Universidad de Santiago de Compostela, 15782 Santiago de Compostela, Spain. Email: alberto.ruano@usc.es.

Background: An integrated care pathway (ICP) is intended to improve the management of prevalent resource-consuming, life-threatening diseases. The purpose of this study was to determine whether the quality of patient care improved with the establishment of a dedicated unit for pulmonary embolism (PE).

Methods: A quasi-experimental pre-post study (pre: years 2010-2013; post: 2015-2020; year 2014, “washing" period) of PE patients $\geq 18$ years (January 2010-June 2020). The intervention involved the implementation of an ICP for PE.

Results: The sample was composed of 1,142 patients (510 pre-intervention and 612 post-intervention) without significant differences between the two populations. In the post-intervention period, significant reductions were observed in the median length of hospital stay (LOS) (8 vs. 6 days); time to start of oral anticoagulation therapy (4.5 vs. 3.5 days; $\mathrm{P}<0.001$ ); and the percentage of patients with high-risk $\mathrm{PE}$ in whom recanalization was not contraindicated $(66.7 \%$ vs. $96 \% ; \mathrm{P}=0.009)$. In-hospital and 30-day mortality decreased, although not significantly (4.5\% vs. $2.8 \% ; \mathrm{P}=0.188 ; 6.1 \%$ vs. $5.2 \% ; \mathrm{P}=0.531$, respectively). Multivariate logistic regression analysis showed that the median LOS intervention decreased significantly according to the service where patients were referred to, and with the use of the simplified PESI. During follow-up, lifelong anticoagulation was prescribed to a higher proportion of patients in the post-intervention period (30.7\% vs. $69.3 \% ; \mathrm{P}<0.001)$.

Conclusions: Although an ICP for PE does not reduce mortality significantly, it improves the quality of patient care.

Keywords: Pulmonary embolism (PE); integrated care pathway (ICP); hospital stay; mortality; anticoagulation

Submitted Apr 05, 2021. Accepted for publication Jul 16, 2021.

doi: $10.21037 /$ jtd-21-595

View this article at: https://dx.doi.org/10.21037/jtd-21-595

^ ORCID: 0000-0001-9927-7453. 


\section{Introduction}

Pulmonary embolism (PE) is a frequent life-threatening clinical entity with an incidence of 1 in 1,000 inhabitants/ year. It is the third most frequent cause of cardiovascular disease and mortality, after myocardial infarction and cerebrovascular disease (1).

PE imposes a considerable social and economic burden. Mortality can only be reduced if clinical practices are improved and the use of diagnostic studies and therapeutical approaches is optimized. Otherwise, adverse events occur resulting in unnecessary costs. Hence the necessity of developing clinical practice guidelines (CPG) and integrated care pathways (ICPs) that ensure the optimization of patient care $(2,3)$. The main objectives of these interventions are improving the effectiveness of clinical processes through coordinated continuous patient care. This approach prevents inconsistencies in clinical practice and, based on scientific evidence, supports the use of more effective processes with the adequate timing, which improves the quality of patient care.

Patient care quality is largely associated with the individual level of variability in clinical decision-making and the multidisciplinary management of a disease (4). In this scenario, an ICP provides the opportunity to establish dedicated units, clinical pathways, rapid pathways and other actions such as the creation of pulmonary embolism response team (PERT) (5-9), unfortunately, at the moment, in our hospital we do not have a PERT.

These interventions are intended to improve the management of prevalent, resource-consuming, lifethreatening diseases such as PE. However, once they are created, their impact on the quality of patient care should be evaluated following the plan-do-check-act sequence (10), which enables planners to quantify improvements. Very few initiatives of this type have been evaluated in Europe, less in Spain.

The purpose of this study is to determine whether the quality of patient care improves with the establishment of an ICP for PE by which emergency care, hospitalization care, and follow-up services are provided by a dedicated unit. Such improvement will be assessed using simple indicators that can be applied in other specialties.

We present the following article in accordance with the STROBE reporting checklist (available at https://dx.doi. org/10.21037/jtd-21-595).

\section{Methods}

\section{Design and approach}

The study was conducted in a tertiary university hospital equipped with over 1,000 beds serving a population of 450,000 . It is a quasi-experimental, pre-post study that included all patients $>18$ years ling admitted to our hospital for PE between January 2010 and June 2020. Patients were recruited by consecutive sampling; all information was collected from electronic health records where the patients were identified with the ICD-9 and ICD-10 diagnostic codes. The intervention involved the implementation of an ICP for PE (supplementary material: https://cdn. amegroups.cn/static/public/jtd-21-595-1.pdf).

Diagnosis of PE was confirmed by helical contrast-enhanced computed tomography scanning of the thorax (11), concurrent to a ventilation/perfusion lung scintigraphy showing a high probability of $\mathrm{PE}$ (according to Prospective Investigation of the Pulmonary Embolism Diagnosis criteria) (12), or the presence of proximal deep vein thrombosis in the lower limbs confirmed on compression ultrasonography in patients with inconclusive findings on ventilation/perfusion scintigraphy (13). Cases were identified by a search of International Classification of Disease, $9^{\text {th }}$ revision codes (ICD-9-CM codes 415.11 and 415.19) and 10th revision codes (ICD-10-CM codes I26.0 and I26.9) on hospital discharge reports. Subjects younger than 18 and patients admitted for causes other than PE who developed $\mathrm{PE}$ as a complication during hospitalization were excluded. Length of hospital stay (LOS) was calculated based on the electronic discharge report.

The study was conducted in accordance with the Declaration of Helsinki (as revised in 2013). The study was approved by the Institutional Review Board of the Health District (registration code 2018/299) and informed consent was taken from all the patients.

\section{Definition of the intervention, pre-post periods and study variables}

After the publication in 2013 of the National Consensus on the Diagnosis, Risk Stratification and Management of patients with PE (14), our Service of Pulmonology designed a specific protocol for the management and follow-up of these patients in a dedicated department of the Service that 
subsequently developed into an ICP for PE. The ICP was designed by a multidisciplinary team composed of specialists from all the services involved in the diagnosis and treatment of the disease. The implementation of the protocol started in 2014, although it was not incorporated into the Intranet of the hospital until 2018 and has been updated after the publication of European Society of Cardiology (ESC) Guidelines for the diagnosis and management of acute PE in 2019 (see supplementary material: https://cdn. amegroups.cn/static/public/jtd-21-595-1.pdf).

The pre-intervention period was the 2010 and 2013 period (both included). 2014 was considered the "washing" period, since it was the first year of implementation. The protocol was not applied for some patients, whereas others were included in the pilot phase of implementation of the protocol. The post-intervention period corresponded to the 2015-2020 period.

\section{Selection of variables}

(I) To compare patient care quality in the two periods, we used the indicators employed by the Spanish Society of Pulmonology and Thoracic Surgery (SEPAR) to grant the certification of excellence in PE care (15), namely: mean hospital stay in patients admitted for PE; percentage of patients with highrisk $\mathrm{PE}$ in whom surgery was not contraindicated, who underwent reperfusion treatment (systemic thrombolysis or percutaneous catheter-directed treatment); in-hospital mortality (percentage of patients admitted for PE who died from $\mathrm{PE}$ in the hospital); major non-fatal bleeding at 30 days; non-fatal recurrences at 30 days; readmission at 30 days; and all-cause mortality at 30 days. Other data included recurrence in patients in whom anticoagulation therapy had been suspended and time from diagnosis to start of oral anticoagulation therapy, where indicated. Other variables were the number of patients admitted to the units of intensive care (ICUs) or respiratory intermediate care units (RICUs) for hemodynamic monitoring and surveillance.

(II) Other data included sex; age; presence of cardiovascular risk factors [smoking, high blood pressure, obesity (body mass index $\geq 30$ ), diabetes, and dyslipidemia]; presence of active cancer; chronic lung disease; heart failure; Charlson index (16) as well as pulmonary embolism severity index simplified version (sPESI) (17); degree of dyspnea (modified from the
Medical Research Council) (18); and time to the start of coumarin anticoagulant therapy (the three last variables are associated with longer LOS) (19).

\section{Statistical analysis}

Univariate analysis was performed to assess differences in the study variables before (pre-intervention, 2010-2013) and after (post-intervention 2014-2020) the dedicated PE Unit was established. Comparisons were carried out by the Chi-square test for categorical variables and for continuous variables, medians comparison test and $t$-student have been used. Significance was set at a $\mathrm{P}$ value $<0.05$. Next, multivariate regression analysis was performed with the intervention period (pre $v s$. post) as the main independent variable. Sex, age, service where the patient was admitted, sPESI and Charlson index were the variables of adjustment. The main independent variable was a hospital stay above or below the median. Results are expressed as Odds Ratio (ORs) with $95 \%$ confidence intervals. All results were analyzed with the IBM SPSS v22 (Armonk, NJ, USA) software package.

\section{Results}

A total of 1,142 patients were included in the study, of whom 510 and 632 had a PE before and after the PE Unit was established, respectively. No relevant sex- or age-based differences were observed as a function of the intervention period. The proportion of never-smokers was slightly higher in the pre-intervention period. No differences were found either in other clinical variables such as dyslipidemia, obesity, hypertension or diabetes, and Charlson index. The proportion of patients classified as intermediate-high risk $\mathrm{PE}$ was slightly higher in the post-intervention period. Table 1 shows the characteristics of the sample by intervention period.

Table 2 compares clinical data related to $\mathrm{PE}$ before and after the Unit was established. There was a significant decrease in the median post-intervention LOS (Figure 1), which decreased from 8 to 6 days. Time from diagnosis to start of oral anticoagulation also decreased from 4.5 to 3.5 days $(\mathrm{P}<0.001)$. The number of patients admitted to the ICU/RICU after the implementation of the ICP increased from $8.2 \%$ to $18.4 \%(\mathrm{P}<0.001)$. The percentage of patients with hemodynamic instability (high risk) was similar in the two periods (pre-intervention: $5.3 \%$ vs. $5.2 \%$ post-intervention). However, the number of reperfusion 
Table 1 Description of pre- and post-intervention samples

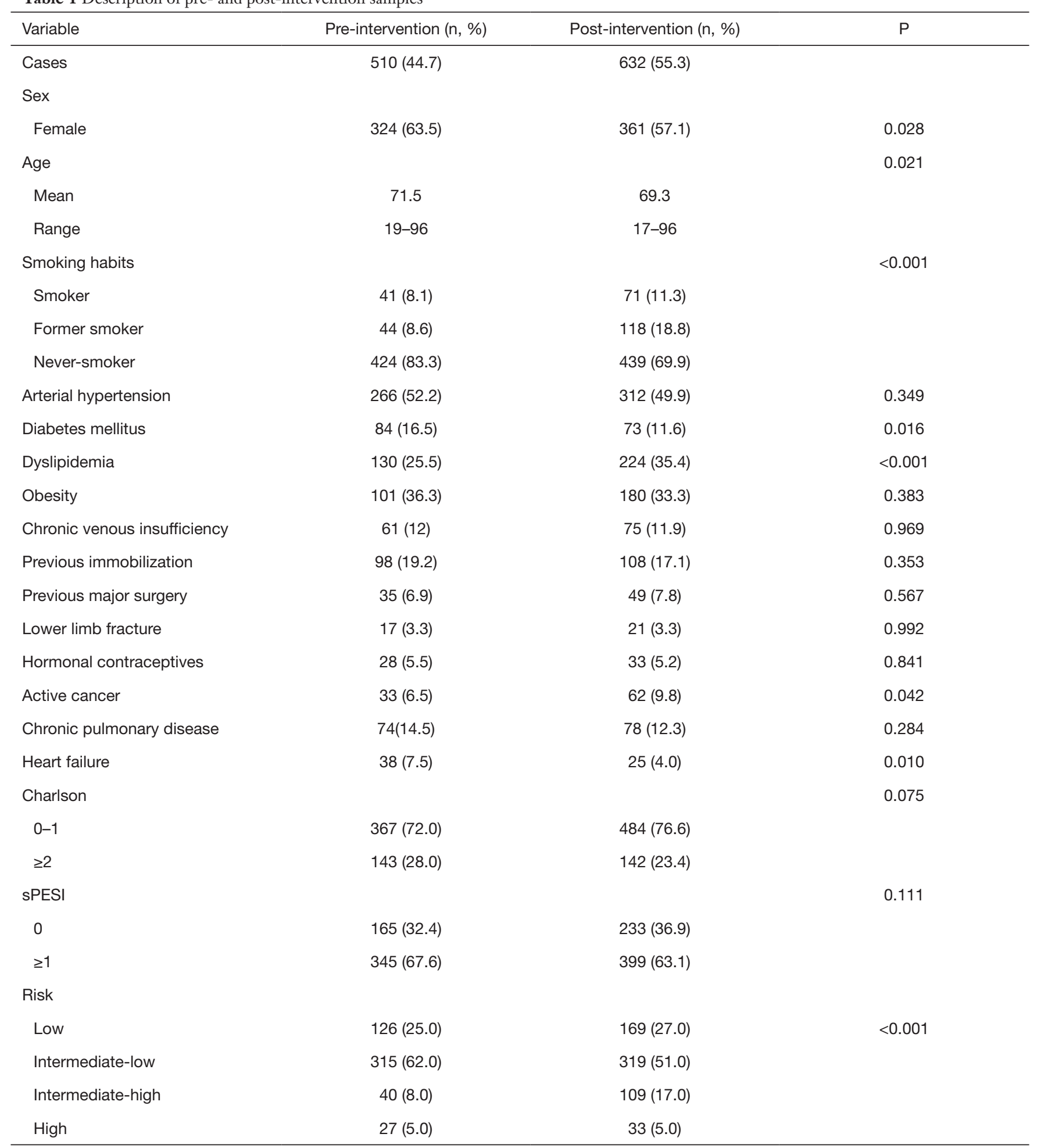

SPESI, simplified pulmonary embolism severity index. 
Table 2 Results of the clinical indicators related to pulmonary embolism before and after the intervention

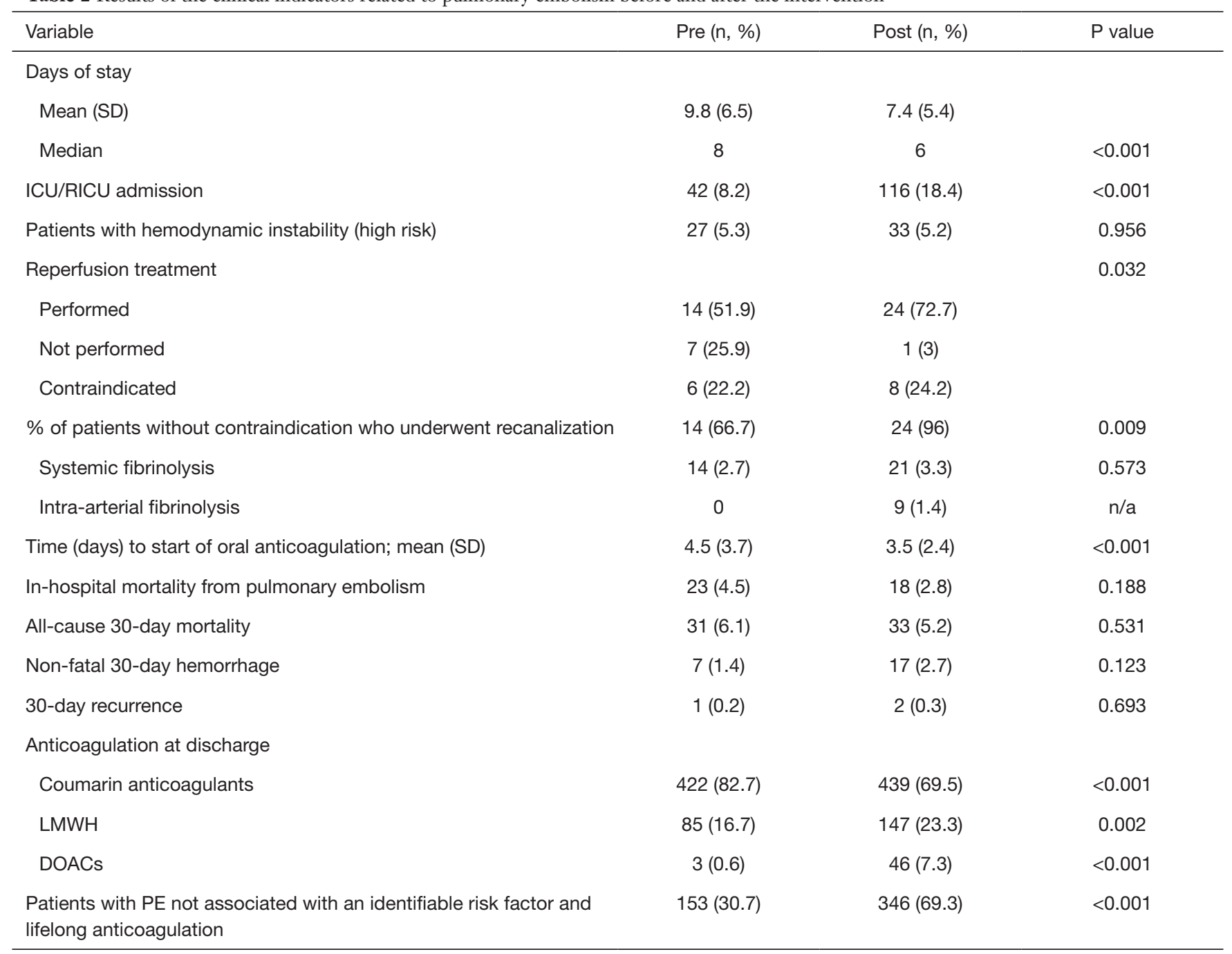

DOACs, direct oral anticoagulants; LMWH, low molecular weight heparin; PE, pulmonary embolism; ICU, intensive care unit; RICU, respiratory intermediate care unit.

treatment and the percentage of patients without contraindication who received this treatment was higher in the post-intervention group $(51.9 \%$ vs. $72.7 \% ; \mathrm{P}=0.032$ and $66.7 \%$ vs. $96 \% ; \mathrm{P}=0.009$, respectively). There was a numerically reduced mortality rate related to $\mathrm{PE}$ before and after the Unit was established, mortality decreased from $4.5 \%$ to $2.8 \%(\mathrm{P}=0.188)$, but it did not reach statistical significance. Mortality at 30 days declined slightly in the post-intervention period, although differences were not significant. The percentage of patients who received lowmolecular weight heparin and direct oral anticoagulants was significantly higher in the post-intervention period (17.7\% vs. $23.3 \% ; \mathrm{P}=0.002$ and $0.6 \%$ vs. $7.3 \% ; \mathrm{P}<0.001$ ); consequently, the proportion of patients who received coumarin anticoagulants was lower $(82.7 \%$ vs. $69.5 \%$; $\mathrm{P}<0.001)$. The proportion of patients with $\mathrm{PE}$ without identifiable risk factors who were prescribed lifelong anticoagulation therapy was significantly lower in the preintervention group (3.7\%) vs. $346(69.3 \%)$ in the postintervention group $(\mathrm{P}<0.001)$.

Table 3 shows the results of multivariate logistic regression, which illustrates the impact of the intervention on the mean LOS adjusted by age, sex, service of admission, and sPESI. The establishment of a dedicated unit reduced significantly the median LOS for PE. Thus, the probability that the LOS exceeded the median halved in the post- 


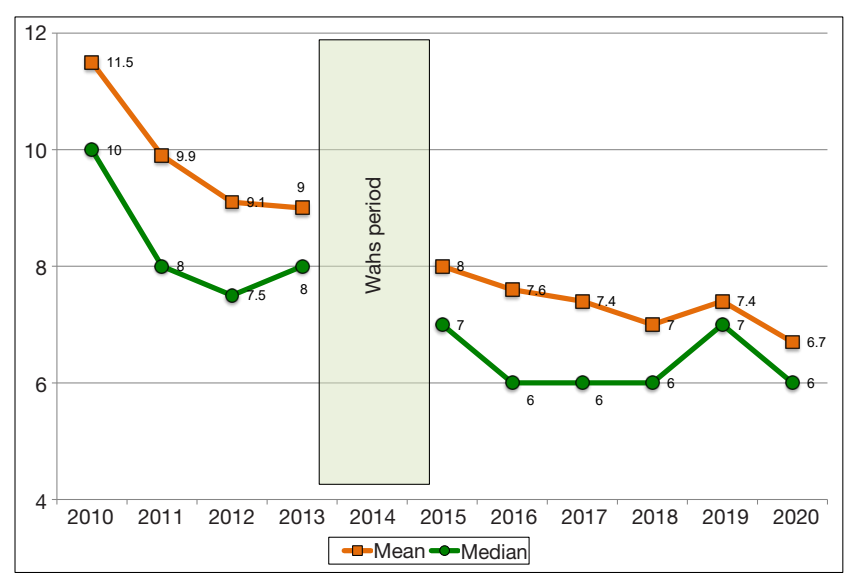

Figure 1 Hospital stay (mean and median) by year. intervention period. Other factors influencing LOS were the service to which the patient was referred after ER admission and sPESI determination.

\section{Discussion}

To the best of our knowledge, this is the first study in Europe to assess the impact of the establishment of a dedicated Unit for Pulmonary on the quality of patient care. The results obtained demonstrate that the establishment of this unit resulted in an improvement in the clinical course of the disease, with a significant reduction of LOS, improved coagulation times, and a reduction (although not significant) of in-hospital mortality. Ambulatory follow-

Table 3 Effect of the different variables on the median length of hospital stay

\begin{tabular}{|c|c|c|c|}
\hline Variable & Raw OR & Adjusted OR & $P$ value \\
\hline Pre-unit & $1(-)$ & $1(-)$ & \\
\hline Post-unit & $0.41(0.32-0.52)$ & $0.56(0.43-0.73)$ & \\
\hline Age & & & 0.620 \\
\hline$\geq 70$ & $1.52(1.19-1.94)$ & $0.93(0.70-1.24)$ & \\
\hline Sex & & & 10.4 \\
\hline Male & $1(-)$ & $1(-)$ & \\
\hline Female & 1.27 (1.00-1.62) & $1.12(0.86-1.45)$ & \\
\hline IM & $8.70(5.23-14.69)$ & $5.19(2.99-8.98)$ & \\
\hline Other & $0.71(0.32-1.57)$ & $0.45(0.19-1.02)$ & \\
\hline sPESI & & & $<0.001$ \\
\hline 0 & $1(-)$ & $1(-)$ & \\
\hline$\geq 1$ & $0.37(0.29-0.48)$ & $2.47(1.83-3.32)$ & \\
\hline Charlson & & & 0.337 \\
\hline $0-1$ & $1(-)$ & $1(-)$ & \\
\hline$\geq 2$ & $1.54(1.18-2-01)$ & $1.16(0.86-1.55)$ & \\
\hline
\end{tabular}

IM, internal medicine; PLM, pulmonology; sPESI, simplified pulmonary embolism severity index; PE, pulmonary embolism. 
up of patients by the dedicated Unit was associated with an increased frequency in the prescription of lifelong anticoagulation therapy in patients with a history of PE. The methods employed are applicable to the evaluation of ICPs in the field of cardiology and pulmonology.

Both, the objective of CPG and ICP for PE is to improve patient care. These tools have a broader scope than PERT, which are intended to form a multidisciplinary team for the design of individualized therapies for acute PE of intermediate-to-high risk. However, no studies have yet been conducted to assess the impact that the establishment of specific units has on the quality of PE management, based on the use of simple, generalizable indicators and using a quasi-experimental, pre-post design.

The difference observed in the number of intermediatehigh risk PE between periods is probably due to the fact that prior to 2014, not as many determinations of troponin levels were carried out. The determination of markers of myocardial damage has made it possible to better classify hemodynamically stable patients who showed data of right ventricular dysfunction on imaging tests. After the intervention, a greater number of patients were monitored, and this implies a better follow-up of the CPGs.

One of the most frequent indicators is the LOS, since hospitalization, along with diagnostic studies and comorbidities of these patients, account for most of the economic burden of PE (20-22). The median LOS in the two periods (pre- and post-intervention) is consistent with the ones reported in our environment (23). During the first year of the pre-intervention period, the median LOS decreased. This reduction may be attributed to the fact that, in 2010, the management of PE patients was assigned to a single specialist to ensure a consistent management of the disease. In the post-intervention period, a significant decrease was found in LOS (8 to 6 days). Hence, the probability that the LOS exceeded the median after the intervention halved in the post-intervention period. Several factors may have influenced these outcomes. First, according to current clinical guidelines, oral anticoagulation must be started the same day of admission, concurrently to parenteral anticoagulation $(1,14,24)$ in order to achieve that the international normalized ratio (INR) is within therapeutic range as fast as possible, without an increase of adverse effects (25). In our case, time to initiation of oral anticoagulation decreased from 4.5 to 3.5 days $(\mathrm{P}<0.001)$, which explains at least partially the reduction in the median LOS. Previous studies have associated the reduction of LOS with early initiation of oral anticoagulation $(26,27)$. On the other hand, according to ESC guidelines, the use of DOACs is preferred over VKA, and this has also been associated with a decrease in hospital stay (28). However, in our study, the majority of our patients receive treatment with VKA despite the fact that DOACs are offered as a first option. The explanation is that our National Health System does not finance the treatment with DOACs for PE and does finance the AVK. Another factor of influence is the service to which the patient was referred after ER admission. A previous study of our group demonstrated that, as compared to referral to the Service of Pulmonology, the OR for a LOS to exceed the median was significantly higher [8.65 (95\% CI: 5.42-13.79)] for patients referred to the Service of Internal Medicine (19). In this study, the data collected in the pre-intervention study confirm previous results (Table 3). Most likely, these differences are explained by a higher adherence to recommendations for the management of PE by the staff of these services $(1,14,24)$ rather than by the fact that patients admitted to the Unit of Internal Medicine may have more comorbidities, as suggested elsewhere (19). This analysis could not be performed in the postintervention period, because since the implementation of the ICP, all patients with a PE were admitted to the unit of Pulmonology. A third factor of influence in LOS is sPESI. In this case, there were no significant differences in the number of patients with a SPESI $\geq 1$ in the two periods.

The percentages of patients who underwent reperfusion treatment or patients with high-risk PE in whom reperfusion treatment was not contraindicated increased significantly in the post-intervention period; all despite a similar number of patients were hemodynamically instable in the two periods. This reflects an improvement in the management of patients with high-risk PE.

In-hospital mortality and 30-day mortality during the two periods were lower than expected (10\%) (29). Of note, both, in-hospital mortality and 30-day mortality decreased in the post-intervention period (in-hospital $4.5 \%$ vs. $2.8 \%$; at 30 days: $6.1 \%$ vs. $5.2 \%$ ), although differences were not significant. Significance may have not been reached due to the low mortality in the two periods. However, other factors may have also caused this reduction, such as the involvement of the Emergency Unit, Intensive Medicine Unit, and Cardiology Unit in the design and implementation of the ICP, or the most strict adherence to CPG for critical care or intermediate care $(8.2 \%$ vs. $18.4 \% ; \mathrm{P}<0.001)$.

The risk of recurrence of thromboembolism was 2.3-2.5 times higher in patients with a non-induced $\mathrm{PE}$ as compared to induced PE (30). For this reason, current CPG suggest 
considering lifelong treatment in patients with $\mathrm{PE}$ without identifiable risk factors, weighing the risk of recurrence against the risk of hemorrhage $(1,14)$. Following this new recommendation, the number of patients with non-induced $\mathrm{PE}$ who were prescribed lifelong anticoagulation therapy in the post-intervention period increased significantly $(69 \%$ vs. $30.7 \% ; \mathrm{P}<0.001)$. This recommendation may result in a reduction of recurrence and an increase in the diagnosis of hidden neoplasms as a consequence of long-term followup. Although these issues are out of the scope of this study, if this criterion would have been applied in the preintervention period, 169 more patients would have been prescribed lifelong anticoagulation therapy. Of them, 17 $(10 \%)$ experienced a relapse. In contrast, during the postintervention period, only one of the 346 patients on lifelong anticoagulation had a relapse after a minimum follow-up of 2 years. This finding is consistent with the results of a meta-analysis that demonstrates that anticoagulation is effective in preventing relapse as long as the treatment is maintained (31). In the post-intervention period, no cases of neoplasm were identified among patients with induced PE vs. 16 cases of neoplasm among patients on lifelong anticoagulation $(4.6 \% ; \mathrm{P}=0.005)$.

The results of this work have some strengths. First, the pre-post design enables comparative analysis of patients seen in the same center, with a similar genetic background, and with a quasi-experimental approach. To avoid distortions during the period of establishment of the Unit, data from year 2014 were excluded from analysis. The sample was relatively large, which allowed us to obtain precise results. Finally, long-term follow-up by the Unit enabled us to determine the proportion of patients who received lifelong anticoagulation therapy.

This study also has some limitations. A randomized clinical trial was not conducted, which would have allowed us to assess the impact of the establishment of the Unit. However, randomization of several health centers and healthy subjects would have been necessary, since, if we assume that the establishment of the Unit was beneficial, it would not have been ethical that a group of patients was treated in other units. Another limitation is that readmissions were not directly analyzed, although this aspect was not within the scope of the study.

Finally, the results of this study demonstrate that the establishment of a PE ICP has a direct impact on patients, as it reduces hospital stay and improves adherence to current recommendations for patients who have an indication for reperfusion treatment. In contrast, a significant reduction in in-hospital mortality was not achieved. These results have an impact on clinical practice, as reducing LOS (mean: 2 days) in a process as prevalent as PE results in a significant reduction of costs and an increased availability of beds for other patients. This type of studies should be conducted to assess the effectiveness of any reorganization in patient care in the field of pulmonology and cardiology.

\section{Acknowledgments}

Funding: None.

\section{Footnote}

Reporting Checklist: The authors have completed the STROBE reporting checklist. Available at https://dx.doi. org/10.21037/jtd-21-595

Data Sharing Statement: Available at https://dx.doi. org/10.21037/jtd-21-595

Peer Review File: Available at https://dx.doi.org/10.21037/ jtd-21-595

Conflicts of Interest: All authors have completed the ICMJE uniform disclosure form (available at https://dx.doi. org/10.21037/jtd-21-595). The authors have no conflicts of interest to declare.

Ethical Statement: The authors are accountable for all aspects of the work in ensuring that questions related to the accuracy or integrity of any part of the work are appropriately investigated and resolved. The study was conducted in accordance with the Declaration of Helsinki (as revised in 2013). The study was approved by the Institutional Review Board of the Health District (registration code 2018/299) and informed consent was taken from all the patients.

Open Access Statement: This is an Open Access article distributed in accordance with the Creative Commons Attribution-NonCommercial-NoDerivs 4.0 International License (CC BY-NC-ND 4.0), which permits the noncommercial replication and distribution of the article with the strict proviso that no changes or edits are made and the original work is properly cited (including links to both the formal publication through the relevant DOI and the license). See: https://creativecommons.org/licenses/by-nc-nd/4.0/. 


\section{References}

1. Konstantinides SV, Meyer G, Becattini C, et al. 2019 ESC Guidelines for the diagnosis and management of acute pulmonary embolism developed in collaboration with the European Respiratory Society (ERS): The Task Force for the diagnosis and management of acute pulmonary embolism of the European Society of Cardiology (ESC). Eur Respir J 2019;54:1901647.

2. Bonfill X, Marzo M. Clinical practice guidelines: highquality and useful. Med Clin (Barc) 2003;120:496-7.

3. Gomis R, Mata Cases M, Mauricio Puente D, et al. Methodological aspects of integrated care pathways. Rev Calid Asist 2017;32:234-9.

4. Grol R, Dalhuijsen J, Thomas S, et al. Attributes of clinical guidelines that influence use of guidelines in general practice: observational study. BMJ 1998;317:858-61.

5. Dudzinski DM, Piazza G. Multidisciplinary Pulmonary Embolism Response Teams. Circulation 2016;133:98-103.

6. Jen WY, Kristanto W, Teo L, et al. Assessing the Impact of a Pulmonary Embolism Response Team and Treatment Protocol on Patients Presenting With Acute Pulmonary Embolism. Heart Lung Circ 2020;29:345-53.

7. Chaudhury P, Gadre SK, Schneider E, et al. Impact of Multidisciplinary Pulmonary Embolism Response Team Availability on Management and Outcomes. Am J Cardiol 2019;124:1465-9.

8. Myc LA, Solanki JN, Barros AJ, et al. Adoption of a dedicated multidisciplinary team is associated with improved survival in acute pulmonary embolism. Respir Res 2020;21:159.

9. Annabathula R, Dugan A, Bhalla V, et al. Value-based assessment of implementing a Pulmonary Embolism Response Team (PERT). J Thromb Thrombolysis 2021;51:217-25.

10. Deming WE. Calidad, Productividad y Competitividad. La Salida de La Crisis. Díaz de Santos, S.A.; 1989.

11. Remy-Jardin M, Remy J, Wattinne L, et al. Central pulmonary thromboembolism: diagnosis with spiral volumetric CT with the single-breath-hold technique-comparison with pulmonary angiography. Radiology 1992;185:381-7.

12. PIOPED Investigators. Value of the ventilation/perfusion scan in acute pulmonary embolism. Results of the prospective investigation of pulmonary embolism diagnosis (PIOPED). JAMA 1990;263:2753-9.

13. Kearon C, Ginsberg JS, Hirsh J. The role of venous ultrasonography in the diagnosis of suspected deep venous thrombosis and pulmonary embolism. Ann Intern Med 1998;129:1044-9.

14. Uresandi F, Monreal M, García-Bragado F, et al. National Consensus on the Diagnosis, Risk Stratification and Treatment of Patients with Pulmonary Embolism. Spanish Society of Pneumology and Thoracic Surgery (SEPAR). Society Española Internal Medicine (SEMI). Spanish Society of Thrombosis and Haemostasis (SETH). Spanish Society of Cardiology (ESC). Spanish Society of Medicine Accident and Emergency (SEMES). Spanish Society of Angiology and Surgery Vascular (SEACV). Arch Bronconeumol 2013;49:534-47.

15. Tabla de criterios para la certificación de la asistencia hospitalaria a los pacientes con EP SEPAR. Available online: https://www.separ.es/node/678

16. Deyo RA, Cherkin DC, Ciol MA. Adapting a clinical comorbidity index for use with ICD-9-CM administrative databases. J Clin Epidemiol 1992;45:613-9.

17. Jiménez D, Aujesky D, Moores L, et al. Simplification of the pulmonary embolism severity index for prognostication in patients with acute symptomatic pulmonary embolism. Arch Intern Med 2010;170:1383-9.

18. Mahler DA, Wells CK. Evaluation of clinical methods for rating dyspnea. Chest 1988;93:580-6.

19. Rodríguez-Núñez N, Ruano-Raviña A, Abelleira R, et al. Factors Influencing Hospital Stay for Pulmonary Embolism. A Cohort Study. Arch Bronconeumol 2017;53:432-6.

20. Motte S, Mélot C, Di Pierdomenico L, et al. Predictors of costs from the hospital perspective of primary pulmonary embolism. Eur Respir J 2016;47:203-11.

21. de Miguel-Díez J, Jiménez-García R, Jiménez D, et al. Trends in hospital admissions for pulmonary embolism in Spain from 2002 to 2011. Eur Respir J 2014;44:942-50.

22. Fanikos J, Rao A, Seger AC, et al. Hospital costs of acute pulmonary embolism. Am J Med 2013;126:127-32.

23. Fernandez MM, Hogue S, Preblick R, et al. Review of the cost of venous thromboembolism. Clinicoecon Outcomes Res 2015;7:451-62.

24. Tritschler T, Kraaijpoel N, Le Gal G, et al. Venous Thromboembolism: Advances in Diagnosis and Treatment. JAMA 2018;320:1583-94.

25. Garcia P, Ruiz W, Loza Munárriz C. Warfarin initiation nomograms for venous thromboembolism. Cochrane Database Syst Rev 2016;(1):CD007699.

26. Qayyum F, Holbrook A, Lam J, et al. Should vitamin $\mathrm{K}$ antagonist therapy be started simultaneously with parenteral anticoagulation: a meta-analysis? Blood Coagul 
Fibrinolysis 2012;23:705-13.

27. Insam C, Méan M, Limacher A, et al. Anticoagulation Management Practices and Outcomes in Elderly Patients with Acute Venous Thromboembolism: A Clinical Research Study. PLoS One 2016;11:e0148348.

28. Paczyńska M, Kurnicka K, Lichodziejewska B, et al. Acute pulmonary embolism treatment with rivaroxaban results in a shorter duration of hospitalisation compared to standard therapy: an academic centre experience. Kardiol Pol 2016;74:650-6.

29. Tagalakis V, Patenaude V, Kahn SR, et al. Incidence of and mortality from venous thromboembolism in a real-

Cite this article as: Rodríguez-Núñez N, Ruano-Raviña A, Lama A, Ferreiro L, Ricoy J, Álvarez-Dobaño JM, SuárezAntelo J, Toubes ME, Rábade C, Golpe A, Riveiro V, Casal A, Abelleira R, González-Barcala FJ, González-Juanatey JR, Valdés L. Evaluation of the impact of an integrated care pathway for pulmonary embolism: a quasi-experimental pre-post study. J Thorac Dis 2021;13(9):5373-5382. doi: 10.21037/jtd-21-595 world population: the Q-VTE Study Cohort. Am J Med 2013;126:832.e13-21.

30. Iorio A, Kearon C, Filippucci E, et al. Risk of recurrence after a first episode of symptomatic venous thromboembolism provoked by a transient risk factor: a systematic review. Arch Intern Med 2010;170:1710-6.

31. van Dongen CJ, Vink R, Hutten BA, et al. The incidence of recurrent venous thromboembolism after treatment with vitamin $\mathrm{K}$ antagonists in relation to time since first event: a meta-analysis. Arch Intern Med 2003;163:1285-93. 